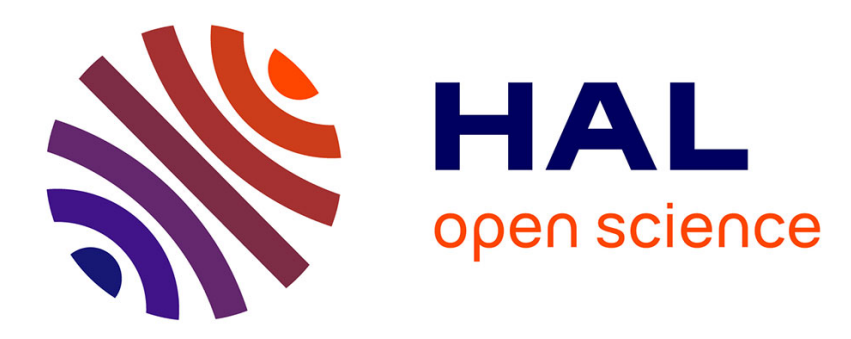

\title{
A new proof of the ultrametric hermite-lindermann theorem
}

\author{
Alain Escassut
}

\section{To cite this version:}

Alain Escassut. A new proof of the ultrametric hermite-lindermann theorem. p-Adic Numbers, Ultrametric Analysis and Applications, 2020, 12 (4), pp.320-323. hal-02891533

\section{HAL Id: hal-02891533 \\ https://hal.uca.fr/hal-02891533}

Submitted on 6 Jul 2020

HAL is a multi-disciplinary open access archive for the deposit and dissemination of scientific research documents, whether they are published or not. The documents may come from teaching and research institutions in France or abroad, or from public or private research centers.
L'archive ouverte pluridisciplinaire HAL, est destinée au dépôt et à la diffusion de documents scientifiques de niveau recherche, publiés ou non, émanant des établissements d'enseignement et de recherche français ou étrangers, des laboratoires publics ou privés. 


\title{
A NEW PROOF OF THE ULTRAMETRIC HERMITE-LINDERMANN THEOREM
}

\author{
ALAIN ESCASSUT
}

\begin{abstract}
We propose a new proof of the Hermite-Lindeman Theorem in an ultrametric field by using classical properties of analytic functions. The proof remains valid in zero residue characteristic.
\end{abstract}

Definitions and notations: The Archimedean absolute value of $\mathbb{C}$ is denoted by $|\cdot|_{\infty}$. Let $\mathbb{K}$ be an algebraically closed complete ultrametric field of characteristic 0 and residue characteristic $p$. We denote by $|$.$| the ultrametric absolute$ value of $\mathbb{K}$ and we denote by $\Omega$ an algebraic closure of $\mathbb{Q}$ in $\mathbb{K}$.

Given $a \in \mathbb{K}$ and $r>0$, we denote by $d\left(a, r^{-}\right)$the disk $\{x \in \mathbb{K}|x-a|<r\}$, we denote by $\mathcal{A}\left(d\left(a, r^{-}\right)\right)$the $\mathbb{K}$-Banach algebra of power series $f(x)=\sum_{n=0}^{\infty} a_{n}(x-a)^{n}$ converging in $d\left(a, r^{-}\right)$and for all $\left.s \in\right] 0, r\left[\right.$, we put $|f|(s)=\sup _{n \in \mathbb{N}}\left|a_{n}\right| r^{n}$. Given $a \in \Omega$, we call denominator of $a$ any strictly positive integer $n$ such that $n a$ is integral over $\mathbb{Z}$ and we denote by $\operatorname{den}(a)$ the smallest denominator of $a$. Next, considering the conjugates $a_{2}, \ldots, a_{n}$ of $a$ over $\mathbb{Q}$ and putting $a_{1}=a$, we put $|\bar{a}|=\max \left\{\left|a_{1}\right|_{\infty}, \ldots,\left|a_{n}\right|_{\infty}\right\}$. Next, we put $s(a)=\log (\max (|\bar{a}|, \operatorname{den}(a))$.

Let $P\left(X_{1}, \ldots, X_{n}\right)=\sum_{j_{1}, \ldots, j_{n}} a_{j_{1}, \ldots, j_{n}} X_{1}^{j_{1}}, \ldots, X_{n}^{j_{n}} \in \Omega\left[X_{1}, \ldots, X_{n}\right]$. We put $|\bar{P}|=$ $\max _{j_{1}, \ldots, j_{n}}\left|\overline{a_{j_{1}, \ldots, j_{n}}}\right|_{\infty}$ and $t(P)=\max \left(\log (|\bar{P}|), 1+\max _{i=1, \ldots, n}\left(\operatorname{deg}_{X_{i}}(P)\right)\right.$.

We denote by $D_{0}$ the disk $d\left(0,1^{-}\right)$and in the case when the residue characteristic of $\mathbb{K}$ is $p>0$ we put $R_{1}=p^{\frac{-1}{p-1}}$ and then we denote by $D_{1}$ the disk $d\left(0, R_{1}^{-}\right)$.

Given a positive real number $a$, we denote by $[a]$ the biggest integer $n$ such that $n \leq a$.

Remark: In particular Levi-Civita's fields have residue characteristic 0 [3].

We aim at proving the Hermite-Lindeman Theorem in an ultrametric field. Our proof is different from the one given by Kurt Mahler in [2] and holds in any ultrametric field, no matter what the residue characteristic. Here, at the beginning, we will follow the methode used in the complex context [4]. But it is impossible to get to the conclusion by considering the zeros of an auxiliary analytic function on only two points, as done in the complex context. So, here we assign an auxiliary function $F_{N}$ to vanish at a number of points roughly equal to $\log (N)$.

\footnotetext{
${ }^{0}$ Keywords: p-adic transcendence numbers, p-adic analytic functions, 1991 Mathematics Subject Classification. Primary 12J25; Secondary 30D35, 30 G06.
} 
Theorem: Suppose that $\mathbb{K}$ has residue characteristic $p>0$ (resp. 0). Let $\alpha \in D_{1}$ be algebraic, (resp. let $\alpha \in D_{0}$ be algebraic). Then $e^{\alpha}$ is transcendental.

Examples: When $p>0, e^{p}, e^{p \sqrt[n]{q}}(n \in \mathbb{N})$ are transcendental.

Let us first recall the following two lemmas:

Lemma 1: Let $a \in \Omega$ be of degree $q$ over $\mathbb{Q}$. Then $-2 q s(a) \leq \log (|a|)$.

Lemma 2 is a particular form of Siegel's Lemma due to M. Mignotte [4]:

Lemma 2: $\quad$ Let $E$ be a finite extension of $\mathbb{Q}$ of degree $q$ and let $\lambda_{i, j} 1 \leq i \leq$ $m, 1 \leq j \leq n$ be elements of $E$ integral over $\mathbb{Z}$. Let $M=\max \left(\overline{\left|\lambda_{i, j}\right|} 1 \leq i \leq\right.$ $m, \mid 1 \leq j \leq n)$ and let $(\mathcal{S})$ be the linear system $\left\{\sum_{j=1}^{n} \lambda_{i, j} x_{j}=0,1 \leq i \leq m\right\}$. There exists solutions $\left(x_{1}, \ldots, x_{n}\right)$ of $(\mathcal{S})$ such that $x_{j} \in \mathbb{Z} \forall j=1, \ldots, n$ and

$$
\log \left(\left|x_{j}\right|_{\infty}\right) \leq \log (M) \frac{q m}{n-q m}+\frac{\log (2)}{2} \forall j=1, \ldots, n .
$$

We can now prove the Theorem.

Proof. We suppose that $\alpha$ and $e^{\alpha}$ are algebraic. Let $h=|\alpha|$. Let $E$ be the field $\mathbb{Q}\left[\alpha, e^{\alpha}\right]$, let $q=[E: \mathbb{Q}]$ and let $w$ be a common denominator of $\alpha$ and $e^{\alpha}$. We will construct a sequence of polynomials $\left(P_{N}(X, Y)\right)_{N \in \mathbb{N}}$ in two variables such that $\operatorname{deg}_{X}\left(P_{N}\right)=\left[\frac{N}{\log (N)}\right], \operatorname{deg}_{Y}\left(P_{N}\right)=\left[(\log N)^{3}\right]$ and such that the function $F_{N}(x)=P_{N}\left(x, e^{x}\right)$ satisfiy further, for every $s=0, \ldots, N-1$ and for every $j=0, \ldots,[\log (N)]$

$$
\frac{d^{s}}{d x^{s}} F_{N}(j \alpha)=0
$$

According to computations in the proof of Hermite Lindeman's Theorem in the complex context, (Theorem 3.1.1 in [4]) we have

$$
\begin{gathered}
\frac{d^{M} F_{N}\left(\gamma_{N}\right)}{d x^{M}}=\sum_{l=0}^{u_{1}(N)} \sum_{m=0}^{u_{2}(N)} b_{l, m, N} \sum_{\sigma=0}^{u_{1}(N)}\left(\frac{u_{1}(N) !}{\sigma !\left(u_{1}(N)-\sigma\right) !}\right)\left(\frac{l !}{\left(u_{1}(N)-\sigma\right) !}\right) m^{u_{1}(N)-\sigma} \\
j^{u_{1}(N)-\sigma} \cdot(\alpha)^{u_{1}(N)-\sigma} \cdot\left(e^{\alpha}\right)^{j u_{2}(N)} .
\end{gathered}
$$

We put $u_{1}(N)=\operatorname{deg}_{X}\left(P_{N}\right), \quad u_{2}(N)=\operatorname{deg}_{Y}\left(P_{N}\right)$. We will solve the system

$$
w^{u_{1}(N)+u_{2}(N)} \frac{d^{s}}{d x^{s}} F_{N}(j \alpha)=0, \quad 0 \leq s \leq N-1, j=0, \ldots,[\log (N)]
$$

where the undeterminates are the coefficients $b_{l, m, N}$ of $P_{N}$. We then write the system under the form

$$
\sum_{l=0}^{u_{1}(N)} \sum_{m=0}^{u_{2}(N)} b_{l, m, N} \sum_{\sigma=0}^{\min (s, l)}\left(\frac{s !}{\sigma !(s-\sigma) !}\right)\left(\frac{l !}{(l-\sigma) !}\right) m^{s-\sigma} \cdot j^{l-\sigma} .
$$




$$
(w \alpha)^{l-\sigma}\left(w e^{\alpha}\right)^{j m} \cdot w^{u_{1}(N)-(l-\sigma)+u_{2}(N)-j m}=0 .
$$

That represents a system of $N[\log (N)]$ equations of at least $N([\log (N)])^{2}$ undeterminates, with coefficients in $E$, integral over $\mathbb{Z}$.

According to computations of Hermite-Lindemann's Theorem in the complex context (Theorem 3.1.1 in [4]), it appears that in the system (2), each term $\left(\frac{s !}{\sigma !(s-\sigma) !}\right),\left(\frac{l !}{(l-\sigma) !}\right), m^{s-\sigma}, \quad j^{l-\sigma},(w \alpha)^{l-\sigma},\left(w e^{\alpha}\right)^{j m}, w^{u_{1}(N)-(l-\sigma)+u_{2}(N)-j m}$ admits a bound of the form $S N(\log (\log (N))$ when $N$ goes to $+\infty$. On one hand $w^{u_{1}(N)+u_{2}(N)}$ is a common denominator and we have

$$
\log \left(w^{u_{1}(N)+u_{2}(N)}\right) \leq \log (\omega)\left(\frac{N}{\log (N)}+\left(\log (N)^{3}\right)\right.
$$

and hence we have a constant $T>0$ such that

$$
\log \left(w^{u_{1}(N)+u_{2}(N)}\right) \leq \frac{T M}{\log M}
$$

Next we notice that

$$
\log \left(\frac{u_{1}(N) !}{\sigma !\left(u_{1}(N)-\sigma\right) !}\right) \leq u_{1}(N) \log \left(u_{1}(N)\right) \leq \frac{N}{\log (N)} \log \left(\frac{N}{\log (N)}\right) \leq N
$$

and similarly,

$$
\log \left(\frac{l !}{\left(u_{1}(N)-\sigma\right) !}\right) \leq u_{1}(N) \log \left(u_{1}(N)\right) \leq N
$$

and

$$
\log \left(m^{u_{1}(N)-\sigma}\right) \leq \frac{3 N}{\log (N)} \log (\log (N)) .
$$

Now, we check that

$\log \left(j^{u_{1}(N)-\sigma} \cdot(|\bar{\alpha}|)^{u_{1}(N)-\sigma} \cdot\left(\left|\overline{e^{\alpha}}\right|\right)^{j u_{2}(N)}\right) \leq N+\frac{N}{\log (N)} \log (|\bar{\alpha}|)+\log (N)(\log (N))^{3} \log \left(\left|\overline{e^{\alpha}}\right|\right)$

and hence there exists a constant $L>0$ such that

$$
\log \left(j^{u_{1}(N)-\sigma} \cdot(|\bar{\alpha}|)^{u_{1}(N)-\sigma} \cdot\left(\left|\overline{e^{\alpha}}\right|\right)^{j u_{2}(N)}\right) \leq L N .
$$

Therefore by (2), (3), (4), (5), (6), (7) we have a constant $C>0$ such that each coefficient $a$ of the system satisfies

$$
s(a) \leq C N(\log (\log (N)) .
$$

By Lemma 2 and by (8) there exist integers $b_{l, m, N}, 0 \leq l \leq u_{1}(N), 0 \leq m \leq$ $u_{2}(N)$ in $\mathbb{Z}$ such that

$0<\max _{l \leq u_{1}(N), m \leq u_{2}(N)} \log \left(\left|b_{l, m, N}\right|_{\infty}\right) \leq \frac{q N \log (N)}{N(\log (N))^{2}-q N \log (N)}(C N \log (\log (N))$

and such that the function

$$
F_{N}(x)=\sum_{l=0}^{u_{1}(N)} \sum_{m=0}^{u_{2}(N)} b_{l, m ; N} x^{l} e^{m x}
$$


satisfies

$$
\frac{d^{s}}{d x^{s}} F_{N}(j \alpha)=0,0 \leq s \leq N-1, j=0,1, \ldots,[\log (N)] .
$$

Now, by (9), we can check that there exists a constant $G>0$ such that

$$
\max _{l \leq u_{1}(N), m \leq u_{2}(N)}\left(\log \left(\left|b_{l, m, N}\right|_{\infty}\right) \leq \frac{G N \log (\log (N))}{\log (N)} .\right.
$$

The function $F_{N}$ we have defined in (10) belongs to $\mathcal{A}\left(D_{1}\right)$ (resp. to $\mathcal{A}\left(D_{0}\right)$ ) and is not identically zero, hence at least one of the numbers $\frac{d^{s}}{d x^{s}} F_{N}(0)$ is not null. Let $M$ be the biggest integer $s$ such that $\frac{d^{s}}{d x^{s}} F_{N}(j \alpha)=0 \forall s=0, \ldots, M-1, j=$ $0,1,2, \ldots,[\log (N)]$. Thus we have $M \geq N$ and there exists $j_{0} \in\{0,1, \ldots,[\log (N)]\}$ such that $\frac{d^{M}}{d x^{M}} F_{N}\left(j_{0} \alpha\right) \neq 0$. We put $\gamma_{N}=\frac{d^{M}}{d x^{M}} F_{N}\left(j_{0} \alpha\right)$.

Let us now give an upper bound of $s\left(\gamma_{N}\right)$. On one hand $w^{u_{1}(N)+u_{2}(N)}$ is a common denominator of all coefficiets and by (2) we have a constant $T>0$ such that

On the other hand, by (1) we have

$$
\log \left(w^{u_{1}(N)+u_{2}(N)}\right) \leq \frac{T M}{\log M} .
$$

$$
\begin{gathered}
\frac{d^{M} F_{N}\left(\gamma_{N}\right)}{d x^{M}}=\sum_{l=0}^{u_{1}(N)} \sum_{m=0}^{u_{2}(N)} b_{l, m, N} \sum_{\sigma=0}^{u_{1}(N)}\left(\frac{u_{1}(N) !}{\sigma !\left(u_{1}(N)-\sigma\right) !}\right)\left(\frac{l !}{\left(u_{1}(N)-\sigma\right) !}\right) m^{u_{1}(N)-\sigma} . \\
j^{u_{1}(N)-\sigma} \cdot(\alpha)^{u_{1}(N)-\sigma} \cdot\left(e^{\alpha}\right)^{j u_{2}(N)} .
\end{gathered}
$$

Now, by (2), (3), (6), (7), (8), (10) and taking into account that the number of terms is bounded by $N(\log N)^{2}$, we can check that there exists a cosntant $B$ such that

$$
s\left(\gamma_{N}\right) \leq B N .
$$

Let us now give an upper bound of $\left|\gamma_{N}\right|$. For convenience, we first suppose that $j_{0}=0$, hence $\frac{d^{M}}{d x^{M}} F_{N}(0) \neq 0$. Set $h=|\alpha|$. Then by Theorem 18.1 in [1] we have $\left|\gamma_{N}\right| \leq \frac{\left|F_{N}\right|(h)}{h^{M}}$. Moreover, we notice that $F_{N}$ admits at least $M[\log (M)]$ zeros in $d(0, h)$ and therefore, by Corollary 22.30 in [1], we have

$\left|F_{N}\right|(h) \leq\left(\frac{h}{R_{1}}\right)^{M[\log (M)]}$ because $\left|F_{N}\right|(r) \leq 1 \forall r<R_{1}$. Consequently, $\left|\gamma_{N}\right| \leq$ $\frac{h^{M(\log (M-1)}}{\left(R_{1}\right)^{M \log M}}$ and hence

$$
\left.\log \left(\left|\gamma_{N}\right|\right) \leq M(\log (M)-1)(\log (h))-M \log (M)\left(\log \left(R_{1}\right)\right)\right) .
$$

Let $\lambda=\log (h)-\log \left(R_{1}\right)$. Then $\lambda<0$. We have $\log \left(\left|\gamma_{N}\right|\right) \leq \lambda M \log (M)-$ $M \log (h)$, hence there exists a constant $A>0$ such that

$$
\log \left(\left|\gamma_{N}\right|\right) \leq-A M \log (M)
$$


Let us now stop assuming that $j_{0}=0$. Putting $z=x-j \alpha$ and $g(z)=f(x)$, since all points $j \alpha$ belong to $d(0, h)$, it is immediate to go back to the case $j_{0}=0$, which confirms (13) in the general case. But now, by Lemma 1, relations (12) and (13) make a contradiction to the relation $-2 q s\left(\gamma_{N}\right) \leq \log \left(\left|\gamma_{N}\right|\right)$ satisfied by algebraic numbers, which shows that $\gamma_{N}$ is transcendental. But then, so is $e^{\alpha}$.

Suppose now that $\mathbb{K}$ has residue characteristic 0 . Then we have to replace $R_{1}$ by $R_{0}$ and the proof works in the same way.

\section{REFERENCES}

[1] Escassut, A. Value Distribution in p-adic Analysis. World Scientific Publishing Co. Pte. Ltd. Singapore, (2015).

[2] Mahler, K. Ein Beweis der Transzendenz der P-adischen Exponentialfunktion, J. reine angew. Math., 169, pp. 61-66 (1932).

[3] Shamseddine, K. A brief survey of the study of power series and analytic functions on the Levi-Civita fields, Contemporary Mathematics, Volume 596, pp. 269-279, (2013).

[4] Waldschmidt, M. Nombres transcendants, Lecture Notes in Mathematics 402, (1974).

${ }^{1}$ Université Clermont Auvergne, UMr CNRS 6620, LMBP, F-63000 ClermontFERRAND, FRANCE.

E-mail address: alain.escassut@uca.fr 